\title{
Microfluidics-enabled phenotyping of a whole population of $C$. elegans worms over their embryonic and post-embryonic development at single-organism resolution
}

\author{
Maria Cristina Letizia', Matteo Cornaglia', Raphaël Trouillon', Vincenzo Sorrentino², Laurent Mouchiroud², \\ Maroun S. Bou Sleiman², Johan Auwerx² and Martin A. M. Gijs ${ }^{1}$
}

\begin{abstract}
The organism Caenorhabditis elegans is a performant model system for studying human biological processes and diseases, but until now all phenome data are produced as population-averaged read-outs. Monitoring of individual responses to drug treatments would however be more informative. Here, a new strategy to track different phenotypic traits of individual C. elegans nematodes throughout their full life-cycle-i.e., embryonic and post-embryonic development, until adulthood onset, differently from life-span-is presented. In an automated fashion, single worms were synchronized, isolated, and cultured from egg to adulthood in a microfluidic device, where their identity was preserved during their whole development. Several phenotypes were monitored and quantified for each animal, resulting in high-content phenome data. Specifically, the method was validated by analyzing the response of C. elegans to doxycycline, an antibiotic fairly well-known to prolong the development and activate mitochondrial stress-response pathways in different species. Interestingly, the obtained extensive single-worm phenome not only confirmed the dramatic doxycycline effect on the worm developmental delay, but more importantly revealed subtle yet severe treatment-dependent phenotypes that are representative of minority subgroups and would have otherwise stayed hidden in an averaged dataset. Such heterogeneous response started during the embryonic development, which makes essential having a dedicated chip that allows including this early developmental stage in the drug assay. Our approach would therefore allow elucidating pharmaceutical or therapeutic responses that so far were still being overlooked.
\end{abstract}

\section{Introduction}

Heterogeneity in response to drugs or treatments can impede the quality of care, thus spurring the development of individualized therapeutic strategies ${ }^{1,2}$. The growing interest in personalized medicine is moving pharmacological research towards the preliminary identification of sub-groups of patients who will benefit from a

\footnotetext{
Correspondence: Martin A. M. Gijs (martin.gijs@epfl.ch)

${ }^{1}$ Ecole Polytechnique Fédérale de Lausanne, Laboratory of Microsystems, Lausanne, Switzerland

${ }^{2}$ Ecole Polytechnique Fédérale de Lausanne, Laboratory of Integrative Systems Physiology, Lausanne, Switzerland
}

specific treatment, from those for whom the treatment will not be effective or even deleterious. Such a priori knowledge would allow tailoring the therapy to the characteristics of each individual, rather than to a population-averaged behavior. To capture the individual variability, longitudinal experiments are needed, where large datasets are collected while maintaining the possibility to observe the response of a single entity. Big data approaches can be applied to identify different subgroups, correlate them with markers and phenotypes and therefore provide new patterns predicting the response to the drug or treatment.

\section{(c) The Author(s) 2018}

(c) (i) Open Access This article is licensed under a Creative Commons Attribution 4.0 International License, which permits use, sharing, adaptation, distribution and reproduction c. in any medium or format, as long as you give appropriate credit to the original author(s) and the source, provide a link to the Creative Commons license, and indicate if changes were made. The images or other third party material in this article are included in the article's Creative Commons license, unless indicated otherwise in a credit line to the material. If material is not included in the article's Creative Commons license and your intended use is not permitted by statutory regulation or exceeds the permitted use, you will need to obtain permission directly from the copyright holder. To view a copy of this license, visit http://creativecommons.org/licenses/by/4.0/. 
Given the cost and high attrition rate of human clinical trials, animal models are still widely used in early stages of drug development to elucidate the therapeutic mechanisms and to extrapolate the drug response to humans. The nematode Caenorhabditis elegans is a popular animal model for the analysis of complex traits, such as metabolism and aging, at the molecular, cellular, organ, and organismal levels ${ }^{3}$. However, the vast majority of the current biological knowledge, for any model, is largely based on population measurements, hiding potentially relevant individual behaviors. In the case of $C$. elegans, the majority of the experimental repertoire is based on analyzing and phenotyping large groups of individuals, hindering the crucial individualized spatiotemporal mapping. Microfluidics has been instrumental in enabling singleworm analysis with robust, high-throughput, and reproducible screenings ${ }^{4-7}$. However, it was not exploited for systematic phenotypic profiling covering embryonic and post-embryonic development of whole single organisms. Authors reported phenotypic platforms, in which cohorts of adult worms were confined in a culture chamber and phenotypic features, such as body size, motility and fluorescence expression, were monitored ${ }^{8}$. However, the lack of single-animal resolution impeded longitudinal phenotypic studies. Single worm isolation was performed either by using droplets, agarose compartments or by confining animals in tapered channels and traps. Animals were encapsulated in droplets starting from the fourth larval (L4) stage $^{9}$ and even from the first larval (L1) stage ${ }^{10}$ and monitored over time, up to worm's death ${ }^{11,12}$. A preliminary study was reported, where eggs were isolated in droplets and new-born worms were kept alive for 4 days in the droplet ${ }^{13}$. However, when performing droplet worm-isolation, delivery of fresh feeding solutions and chemicals is still challenging. Similarly, larvae were manually isolated in agarose micro-compartments and then cultured $^{14,15}$. This approach still requires long manual preparatory steps and lacks the possibility of active fluidic exchange, crucial for automation purposes. A few screenings on single animals have been shown, where L4 or adult worms were temporarily confined in customized channels, while short behavioral and anatomical features were observed and measured ${ }^{16-18}$. Also longitudinal observations on single worms were performed. To this purpose, previously synchronized populations of adult worms or larvae were synchronized offchip and injected into the device, isolated in tapered channels or by multilayer pressure valves, before being confined in a culture chamber. Different phenotypes were monitored, such as worm size, motility, progeny number, mating and development time $\mathrm{e}^{16,19-21}$. However, in these cases worm synchronization was performed either by manual and time-consuming synchronizations off-chip or by using aggressive bleaching steps that could compromise the integrity of the organism. Moreover, typically, these analysis were performed starting from the L4 stage of the worms, thus preventing monitoring phenotypic variations occurring during worm embryonic and post-embryonic development. Studies were reported, where hydrodynamic or dielectrophoretic forces were exploited for long term trapping and imaging of zebrafish or nematode Panagrolaimus davidi embryos on chip ${ }^{22-24}$. Recently, a protocol to automatically collect and isolate embryo directly from egg laying on-chip, avoiding bleaching or manual procedures, and monitor embryonic development was shown ${ }^{25}$. However, a comprehensive quantitative and dynamical analysis of phenotypes in single $C$. elegans from embryo to adulthood was not performed yet. It is clear that a platform allowing singleworm based multi-phenotypic measures upon a specific treatment for the entire life-cycle would pave the way for extensive high-content, individualized analysis and possibly targeted follow-up treatments.

In this context, this article describes an innovative chipbased strategy for the precise analysis of the heterogeneous response to pharmacological treatments-and the resulting identification of heterogeneous biomarkers or phenotypes-amongst a controlled C. elegans population over the embryonic and larval development. In this endeavor, a microfluidic platform was designed to automatically isolate naturally-synchronized embryos upon egg laying and culture them into single-worm chambers, where the identity of each organism was preserved during its life-cycle. Several phenotypes were monitored for each worm, resulting in high-content phenome data for each experimental condition. Once all the individual phenotypes were extracted, one could match the different experimental conditions with phenotypic signatures. Multivariate methods were employed to navigate the datasets and identify only the most important phenotypes for each experimental condition. We validated our method by analyzing the response of $C$. elegans to doxycycline, an antibiotic known for extending the life-span of different experimental models and for activating the mitochondrial unfolded protein response $\left(U P R^{\mathrm{mt}}\right)$, a stress-response that is conserved across species ${ }^{26-28}$. $\mathrm{UPR}^{\mathrm{mt}}$ induction during larval development in C. elegans leads to reduced mitochondrial function ${ }^{26,29}$, as well as developmental delay and decreased brood size ${ }^{26,29,30}$. However, the relevance of these phenotypes to the lifespan extension, as well as their relative variations after $\mathrm{UPR}^{\mathrm{mt}}$ induction, is still unclear. The single-worm-based analysis method was therefore employed to examine these treatment-dependent phenotypes. Interestingly, the population-averaged response to doxycycline matched previous results confirming the doxycycline-dependent extension of the worms' life-cycle duration. However, more importantly our single-worm-based phenotyping 
a

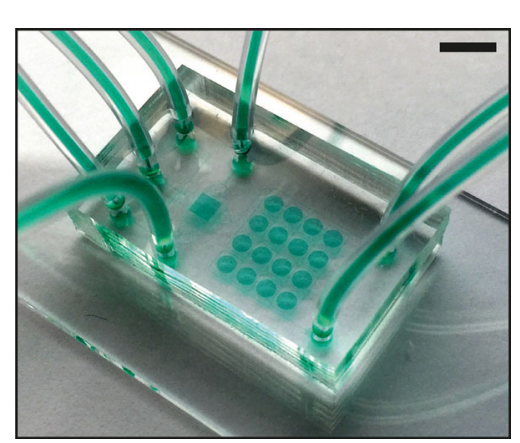

C

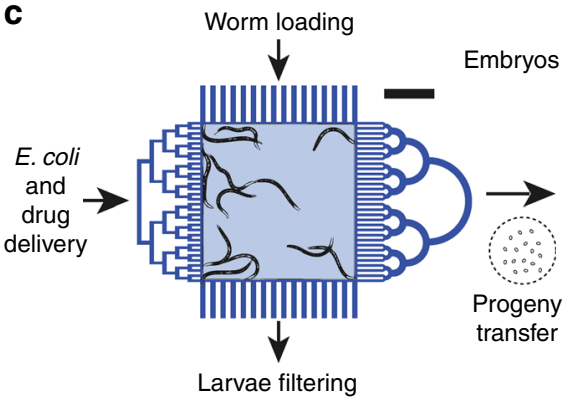

b

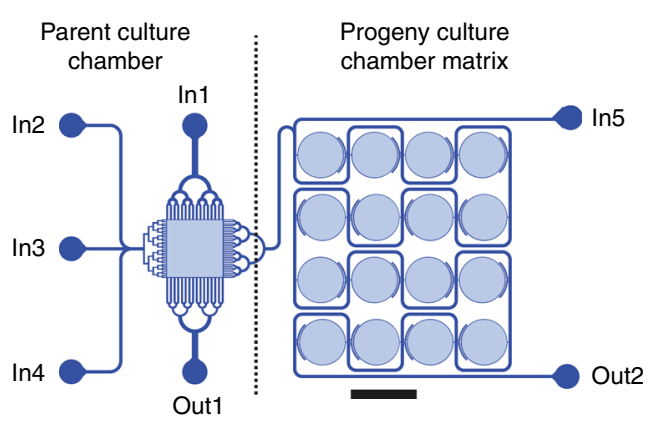

d

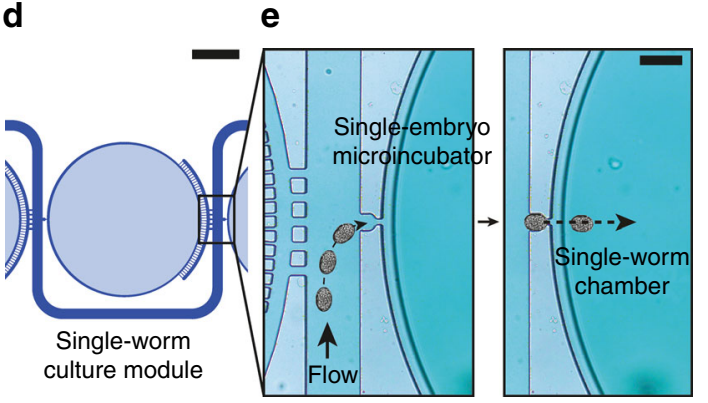

Fig. 1 Automated platform for isolation and monitoring of single C. elegans organisms during full life-cycle. a Picture of the microfluidic device. Scale bar $=5 \mathrm{~mm}$. $\mathbf{b}$ Schematic of the microfluidic device, showing the parent culture chamber and the progeny culture chamber matrix. Scale bar $=2 \mathrm{~mm}$. c Zoom on the parent culture chamber including typical drawings of young adult C. elegans. Scale bar $=500 \mu \mathrm{m}$. d Schematic representation of a module of the progeny culture chamber matrix. Scale bar $=500 \mu \mathrm{m}$. e Sketch of the egg hydrodynamic trapping in a singleembryo micro-incubator and its transfer in the adjacent single-worm chamber. Scale bar $=100 \mu \mathrm{m}$

analysis additionally revealed subtle but significant responses characteristic of sub-groups of animals, observed during both the embryonic and the larval development, and that would have not be spotted using a different device and phenotyping approach.

\section{Materials and methods}

\section{Fabrication of the microfluidic chips}

Microfluidic chips were prepared by soft lithography ${ }^{31}$ using 2-layer SU-8 on a silicon mold. First, the mold was fabricated by traditional photolithography. After fabricating a $40 \mu \mathrm{m}$-thick SU-8 pattern on a silicon 4 -inch wafer, the second $90 \mu \mathrm{m}$-thick SU-8 layer was patterned on top of the first. Secondly, such a mold was used for polydimethylsiloxane (PDMS) casting. We poured a liquid PDMS mixture with a weight ratio base:curing agent of $10: 1$, waited for degassing and cured at $100{ }^{\circ} \mathrm{C}$ for $1 \mathrm{~h}$. Thirdly, after de-molding, we diced each PDMS 13 $\mathrm{mm} \times 23 \mathrm{~mm}$ chip, and punched holes into the PDMS to form inlets and outlets, and bonded each to a $26 \mathrm{~mm} \times 76$ $\mathrm{mm}$ glass slide, upon air-plasma surface activation. Finally, tubings were plugged in the PDMS holes. A picture of the final glass-PDMS microfluidic device is given in Fig. 1a.

\section{Platform control and image acquisition}

The microfluidic chip was integrated onto an inverted microscope (Axio Observer, Zeiss) equipped with a digital camera (Hamamatsu, Japan) and two illumination systems: (i) a precisExcite High-Power LED Illumination system (Visitron, Puchheim, Germany) for brightfield imaging and (ii) a Lambda DG4 illumination system (Sutter instruments, Novato, CA, USA) for fluorescence imaging. The microscope was equipped with a motorized xyz-stage that had an ASI piezo controller for z-displacement (Visitron, Puchheim, Germany) and the automated imaging process was controlled using VisiView Premier Image acquisition software (Visitron, Puchheim, Germany). To start the automated imaging process, we initialized the position of either each embryo-incubator or each single-worm chamber as single point of the xyz-stage scanning. We also programmed the acquisition system so that brightfield (exposure time $=2 \mathrm{~ms}$ ) and fluorescent (exposure time $=150 \mathrm{~ms}$ ) pictures were recorded every 7 min on each position. A Zeiss $20 \times$ NA 0.22 objective was used for imaging of single-embryos in the micro-incubator, while a Zeiss $4 \times$ NA 0.075 objective was used for imaging of each single-worm chamber. A single picture was taken to capture each worm at a time-point, as the 
field of view of the objective was large enough to accommodate the whole single-worm chamber. An immobilization method was not needed, as the mobility of the worms was low. Indeed, the presence of optimized amounts of food along the duration of the whole experiment limited food-seeking behavior and motion. To capture sharp fluorescent pictures, the exposure time was finely tuned to avoid extensive animal movement within the exposure time window. The fluorescent picture was captured $150 \mathrm{~ms}$ (fluorescent exposure time) after the brightfield picture. Afterwards, the motorized stage moved to the following single-worm chamber and waited $3 \mathrm{~s}$ for stabilization, before brightfield and fluorescent pictures were shot. In this way, we could image the full array in about $50 \mathrm{~s}$. Considering the typical timescale of the evolution of the development of the worms $(\sim 10 \mathrm{~h}$ for embryonal development and 2-5 days for larval development), this imaging routine is not expected to introduce a development mismatch between the different worms. The microfluidic operations were controlled using Nemesys syringe pumps (Cetoni, Korbussen, Germany). Given the relatively long time scale of each experiment, the syringe dedicated to nutrient Escherichia coli $(E$. coli) delivery was provided with a custom-made stirring system in order to prevent bacteria from sedimenting at the bottom of the syringe. We placed a magnetic $\operatorname{rod}(1 \mathrm{~mm} \times 1 \mathrm{~mm} \times 2 \mathrm{~mm})$ (Supermagnete, Uster, Switzerland) inside the syringe. A rigid tube with small magnet rods blocked inside and fixed to the axis of a DC motor $6 / 9 \mathrm{~V}$ (Arduino, Ivrea, Italy) was positioned adjacent to the syringe. The rotation of the motor axis and its rods induced agitation in the magnet inside the syringe. The rotation of the motor axis was controlled by a microcontroller (Arduino, Ivrea, Italy) and it was synchronized to the syringe control, so that $E$. coli was stirred before being injected into the microfluidic system.

\section{Microfluidic platform operation}

A schematic of the microfluidic device is reported in Fig. 1b. The microfluidic chip and tubing were first filled with a solution of Pluronic F-127 injected from inlet In2 and incubated overnight, in order to prevent E. coli sticking and clogging inside the microfluidic channels ${ }^{32}$. A mixed population of worms was suspended in $200 \mu \mathrm{L}$ of S medium and sucked up in a tube connected to the device. Afterwards, with a flow of $500 \mathrm{~nL} / \mathrm{s}$, worms were injected in the microfluidic chip from inlet In1 in the parent culture chamber, where they were automatically synchronized. Outlet Out1 was provided with tailored filters, designed in such a way that only young adult (YA) and adult worms were retained in the chamber, while younger worms were washed out by the flow. The number of worms retained in the chamber was controlled by the concentration of YA and adults (typically from 5 to 10 worms) in the $200 \mu \mathrm{L}$ solution. After worm synchronization, outlet Out 1 was closed, while Out 2 was opened. Worms were then exposed to a constant flow of fresh $\mathrm{S}$ medium at $15 \mathrm{~nL} / \mathrm{s}$ from inlet In3, and a constant flow of $20 \mathrm{~nL} / \mathrm{s}$ of $E$. coli bacteria-containing treatment when needed-from inlet In4. With this flow program, we not only provided medium and nutrients to the parental population until worms were ready for egg laying, but also automatically and simultaneously delivered freshly-laid embryos towards the progeny culture chamber matrix. In the doxycycline experiment, embryos were treated with a solution of doxycycline in E. coli provided from inlet In5. Once the full array of single-embryo micro-incubator was filled and the embryos reached the twitching-onset stage, we simultaneously transferred all the embryos from each incubator to the adjacent single-worm chamber, by applying a flow of $\mathrm{S}$ medium at $20 \mu \mathrm{L} / \mathrm{s}$ for $2 \mathrm{~s}$ from inlet In3, taking advantage of PDMS elasticity. After the embryo transfer, the parental generation was removed from the chip. To do so, outlet Out1 was opened and a flow of $20 \mu \mathrm{L} / \mathrm{s}$ was applied from inlet In1 until all the adult worms were washed out. Afterwards, we closed outlet Out1 as well as inlets In1, In2, In3, and In4. From this step on, only inlet In 5 and outlet Out 2 were used. A flow profile adapted to larval age was developed, in order to provide enough nutrients to the progeny generation (See Supplementary Fig. S1). During the embryonic twitching-hatching stage and during the larval 1 (L1) stage, we delivered a repetitive loop flow profile of $5 \mathrm{~nL} / \mathrm{s}$ for $10 \mathrm{~min}$ and $20 \mathrm{~nL} / \mathrm{s}$ for $15 \mathrm{~s}$, in order to ensure that the newborn larvae could not escape through the singleembryo micro-incubator, as the incubator aperture dimension matched the L1 size. Afterwards, a loop flow profile of $10 \mathrm{~nL} / \mathrm{s}$ for $15 \mathrm{~min}, 150 \mathrm{~nL} / \mathrm{s}$ for $15 \mathrm{~s}$ and $0 \mathrm{~nL} / \mathrm{s}$ for $15 \mathrm{~min}$ was used, in order to refresh $E$. coli bacteria in the chambers, to avoid stressing the worms with continuous hydrodynamic forces. The experiments were performed at $25^{\circ} \mathrm{C}$.

\section{Chemicals and materials}

Four-inch $550 \mu \mathrm{m}$ thick Si wafers, 5-inch chromiumglass masks, and de-ionized water (DIW) were obtained from the Center of Microtechnology and Nanotechnology at EPFL. GM 1070 SU-8 negative photoresist was purchased from Gersteltec (Pully, Switzerland). PDMS Sylgard 184 was acquired from Dow Corning (Wiesbaden, Germany). One milliliter polycarbonate syringes were purchased from BD (Franklin Lakes, NJ USA). Tygon tube with $0.51 \mathrm{~mm}$ inner and $1.52 \mathrm{~mm}$ outer diameters was bought from Fisher Scientific (Wohlen, Switzerland). Pluronic F-127 was purchased from Sigma-Aldrich (Buchs, Switzerland). S medium buffer was obtained by adding $1 \mathrm{~L} \mathrm{~S}$ Basal, $10 \mathrm{~mL} 1 \mathrm{M}$ potassium citrate $\mathrm{pH}$ 6, 10 
$\mathrm{mL}$ trace metals solution, $3 \mathrm{~mL} 1 \mathrm{M} \mathrm{CaCl}, 3 \mathrm{~mL} 1 \mathrm{M}$ MgSO. Pluronic F-127 solution was prepared by diluting 0.4\% (weight/volume) Pluronic F-127 in DIW. Doxycycline (Sigma), was dissolved in water to a stock concentration of $6 \mathrm{mg} / \mathrm{mL}$.

\section{Image processing}

Image processing was performed with ImageJ software (https://imagej.nih.gov/ij/, RRID = SCR_003070) and Matlab R 2015b software (MathWorks, U.S.A, RRID = SCR_001622), on each stack of brightfield and fluorescent worm images. Brightfield pictures were processed in order to spot bean stage onset, twitching onset, hatching, molting times and appearance of protruded vulva for each individual. The number of surviving embryos was assessed by counting the number of hatching eggs. For each larval stage, length and diameter (or width, as the worms are assumed cylindrical) were calculated and averaged over five brightfield pictures at the center of the larval stage for each individual. In order to assess fertility, we looked for the presence of internal embryos from self-fertile reproduction after vulva protrusion. To extract fluorescent signal-to-background (SBR) ratio, the region of interest (ROI) corresponding to the worm area was first segmented in the whole stack. We performed background subtraction and applied a median filter in order to remove salt-and-pepper noise. SBR was then calculated as SBR = $\left(\mathrm{I}_{\mathrm{ROI}}{ }^{-} \mathrm{I}_{\mathrm{BG}}\right) / \mathrm{I}_{\mathrm{BG}}$ for each picture, with $\mathrm{I}_{\mathrm{ROI}}$ average intensity of the signal in the ROI and $\mathrm{I}_{\mathrm{BG}}$ the average intensity of the background. In order to remove noise and artifacts from the obtained SBR, a moving average filter (length of moving window $=10$ frames) was applied on the stack.

\section{Experimental model and subject details}

C. elegans strains were cultured at $20^{\circ} \mathrm{C}$ on nematode growth media (NGM) $90 \mathrm{~mm}$ Petri dishes seeded with $E$. coli strain OP50 (RRID = WB-STRAIN:OP50). The strain used in this study was the SJ4100 (zcIs13[hsp6p::GFP] RRID = WB-STRAIN:SJ4100), provided by the Caenorhabditis Genetics Center (University of Minnesota). For studies in untreated conditions, hsp-6p::gfp worms were plated on regular NGM plates at the L4 stage, and collected about $12 \mathrm{~h}$ later at the stage of YA for injection in the system. For the doxycycline treatment, a concentration of $30 \mu \mathrm{g} / \mathrm{mL}$ was chosen to trigger $\mathrm{UPR}^{\mathrm{mt}}$ in $h s p-6 p:: g f p$ worms, as previously described on nematode growth medium $(\mathrm{NGM})^{29,33}$. Adult gravid worms were placed on control plates and removed after $6 \mathrm{~h}$ after egg laying. F1 YA worms were then collected for introduction in the microfluidics system, where the treatment with the antibiotic $(30 \mu \mathrm{g} / \mathrm{mL})$ was initiated after egg laying. Worms were suspended in $\mathrm{S}$ medium solution prior to each microfluidic experiment.

\section{E. coli culture}

HT115 E. coli bacteria (RRID = WB-STRAIN:HT115) were grown in Luria Broth (LB) with $100 \mu \mathrm{g} / \mathrm{mL}$ ampicillin and $12.5 \mu \mathrm{g} / \mathrm{mL}$ tetracycline overnight in a thermal shaker at $37^{\circ} \mathrm{C}$. The following day, $50 \mu \mathrm{L}$ of the confluent bacterial cultures were used to inoculate freshly prepared LB medium containing only ampicillin, so to avoid the presence of tetracycline during the worm experiments, which has been shown to also affect mitochondrial function, similarly to doxycycline ${ }^{33}$. The new cultures were grown until reaching an optical density between 0.6 and 0.8 , and $90 \mu \mathrm{L}$ were used for seeding the experimental plates.

\section{Quantification and statistical analysis}

The on-chip analysis provided a multitude of phenotypic variables which precluded the identification of major phenotypic changes in response to the experimental conditions. As a consequence, we used a hierarchical cluster analysis and principal component analysis (PCA) (see $\mathrm{SI}$ ) to reduce the dimensionality of the datasets and emphasize the driving force that generated the raw data.

The input experimental variables were the durations of the embryonal stages of development, the durations of the five stages of larval development, the length at the L1 stage and length increments at the other four stages of larval development, and the fluorescent SBR at L1 stage and the SBR increments at the other four stages of larval development. Increments, rather than absolute measurements, were used here to minimize the risk of unwanted correlations between parameters which are expected to increase as the worm develops (for instance the length), which may hide smaller variations between the treatments. Each dataset was normalized and centered to the average of each parameter for the negative control, and a hierarchical cluster analysis was run using the built-in algorithm in Matlab R 2015b (MathWorks, USA, RRID = SCR_001622). Data points were organized in clusters ordered according to the unweighted average Euclidean distance. Statistical analysis was performed using GraphPad Prism (San Diego, CA, USA, RRID = SCR_002798). Graphs are expressed as mean \pm SD overlaid with a cloud of points describing the actual dataset, unless otherwise indicated in the figure legends. For each treatment, the datasets were independently obtained from two chips, each featuring 16 worm chambers. The sample number $(\mathrm{N})$ indicates the number of independent viable biological samples for which parameters were extracted in each dataset. Statistical significance was determined with a two-tailed Mann-Whitney test. $p$-values were graphically reported as * $p \leq 0.05, " * p \leq 0.01$. Untreated $h s p-6 p:: g f p$ worms are used as controls. Statistical tests were performed only to compare the recorded values of a given phenotypic parameter, at a specific developmental stage, amongst different treatments/groups of worms. 


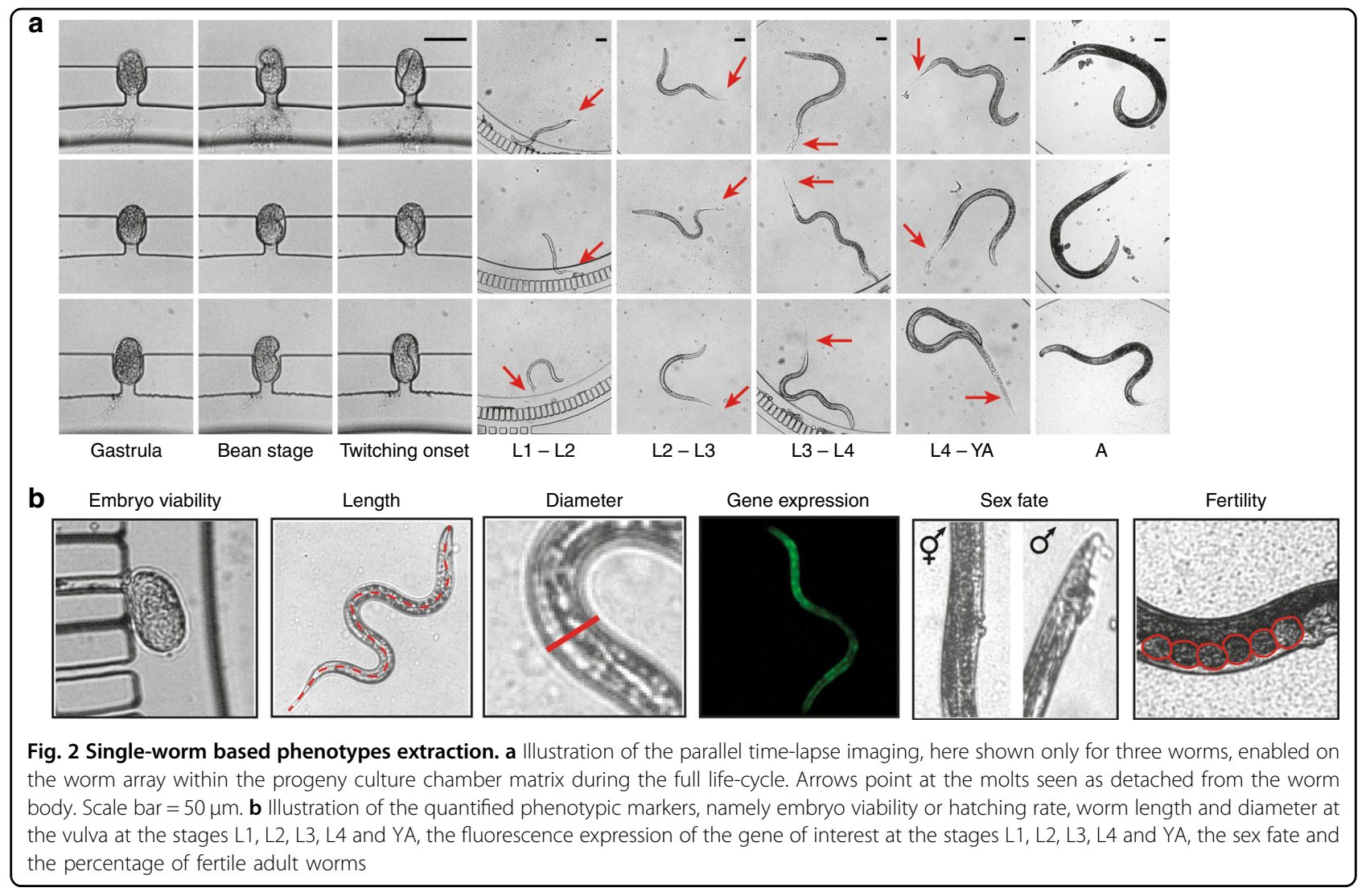

\section{Results}

Single-worm high-content phenotypic platform

A microfluidic chip for the phenotypical analysis and biomarker identification at single-worm level throughout its life-cycle was designed and fabricated in polydimethylsiloxane (PDMS) and glass (Fig. 1a). First, a nonsynchronized population of $C$. elegans was loaded in the parent culture chamber (Fig. 1b, c). In order to synchronize the population in an automated way, dedicated filters were fitted to outlet Out1 to retain only young adult (YA) worms in the chamber and wash younger worms out. Afterwards, those YA worms were fed with $E$. coli bacteria until egg production. Once laid, embryos were transported by the flow to the progeny culture chamber matrix (Fig. 1b, d), consisting of an array of 16 single-worm chambers, each fitted with a trap for single-embryo capture (Fig. 1e). Through hydrodynamic trapping the embryos occupied consecutive traps, forming a naturallysynchronized population $^{25}$. More details about the design of the hydrodynamic trapping system are provided in the SI. Using this approach, the traditional aggressive bleaching step and the time consuming manual synchronization procedures were avoided $^{34}$. The singleembryo traps were presented and discussed in a previous study published by our group ${ }^{25}$. The device used in the current work pushes further this concept by connecting a worm-culture chamber to each singleembryo trap (Fig. 1d, e). With this approach, the newborn larva remains isolated and can be cultured until adulthood onset, allowing for the first time the on chip monitoring of embryonic and post-embryonic development. In fact, after capture, each embryo was simultaneously injected from the micro-incubator to the adjacent single-worm chamber by increasing the flow rate, exploiting PDMS elasticity (Fig. 1e). Brightfield and fluorescent pictures were automatically taken at a fixed rate, first of each single-embryo micro-incubator and then of each single-worm chamber, from egg laying until adulthood onset. This guaranteed sufficient temporal resolution to describe transitions between the different development stages.

We identified the milestones characterizing embryonic and larval development ${ }^{35,36}$, detailed in Fig. 2a. C. elegans embryos, laid upon beginning of gastrulation, were immediately trapped in the embryo-incubator array. Bean stage and twitching onset were observed in the singleembryo incubator, while egg hatching was detected in the single-worm chamber. The duration of each larval stage could be unambiguously evaluated by measuring the time interval between consecutive moltings, which identify the actual biological transitions amongst the different larval stages ( $\mathrm{L} 1$ to L4), up to the YA stage. The molting events 


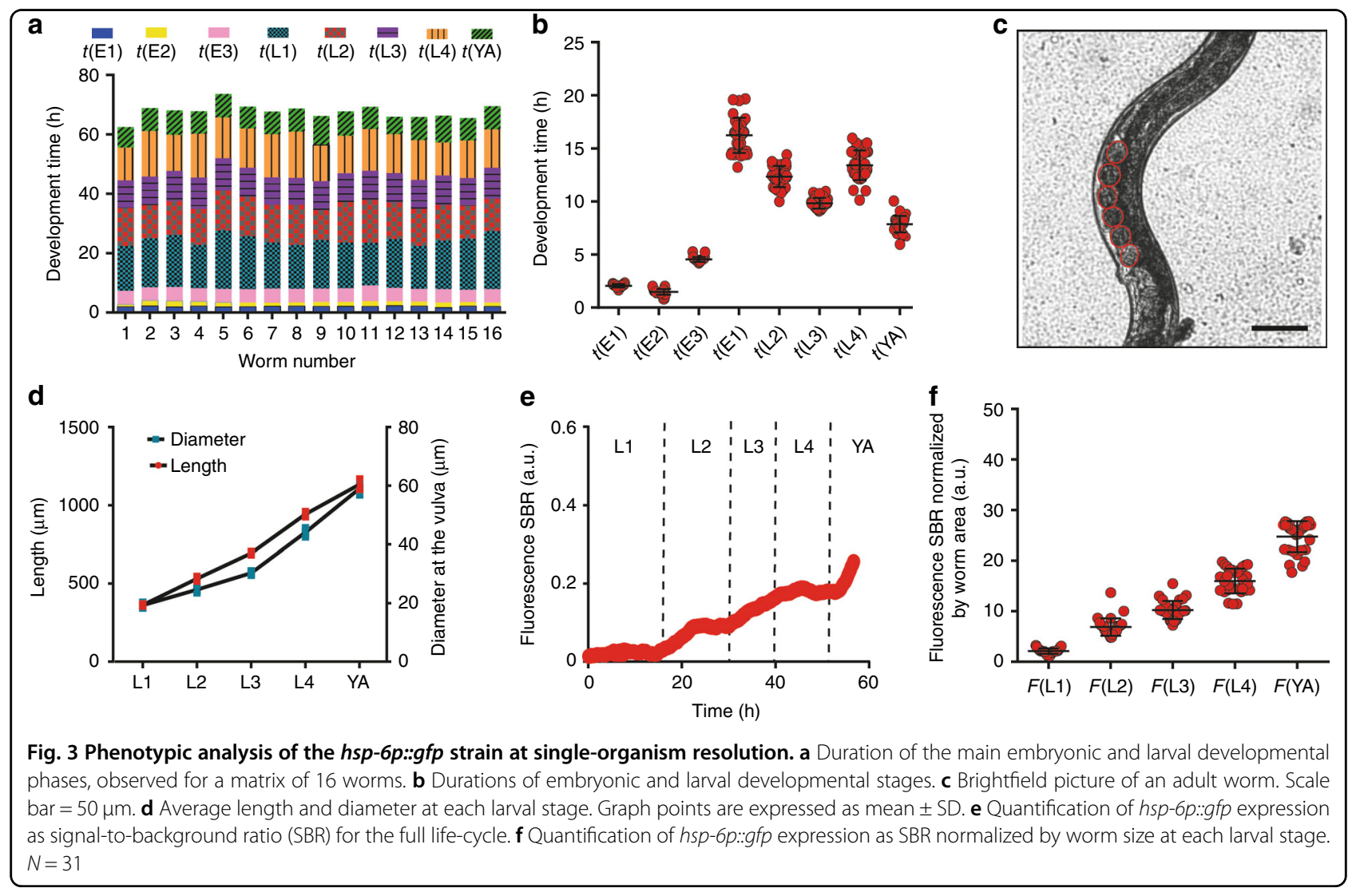

are the perfect indicators of the larval development status and spotting all the four moltings of each animal represents a unique feature of our single-worm culture chip. Adulthood onset was defined by the vulva protrusion, which normally coincides with the appearance of first selffertilized embryos inside the worms, and which determined the end of the life-cycle and therefore our experiment. Other important phenotypes were extracted for each larval stage of development, for each animal (Fig. 2b): the hatching rate, the average body length and diameter at the vulva, and the expression level of a gene of interest via fluorescent read-out. Moreover, the sex fate (hermaphrodite/male) and the fertility rate were recorded as well.

\section{Phenotypic biomarker analysis of a control strain}

High-content phenotypic data on $h s p-6 p:: g f p$ worms, a nematode $\mathrm{UPR}^{\mathrm{mt}}$ reporter strain ${ }^{37}$ that can be considered, except for the fluorescent expression, equivalent to N2 wildtype strain, was obtained by extracting biologicallyinformative phenotypic markers from the images. The durations of the developmental stages along the life-cycle - gastrula-bean stage $t(E 1)$, bean stage-twitching $t(E 2)$, twitching-hatching $t(E 3)$, larval stages $t(L 1), t(L 2), t(L 3)$, $t(L 4)$ and $t(Y A)$ stage-were systematically quantified for individual worms (16 per array, Fig. 3a) and averaged over the array (Fig. 3b). In the absence of treatment, the duration of these stages matched those reported for worms cultured on agar plates at the same temperature ${ }^{38}$. Moreover, $97 \%$ of the trapped embryos developed and $100 \%$ of them became fertile hermaphrodite adults, as internal eggs were observed (Fig. 3c). The average worm lengths, corresponding to the skeleton (later abbreviated as $\mathrm{L}(\mathrm{L} 1), \mathrm{L}(\mathrm{L} 2), \mathrm{L}(\mathrm{L} 3), \mathrm{L}(\mathrm{L} 4)$ and $\mathrm{L}(\mathrm{YA})$ ), and diameters, at each larval stage were also obtained (Fig. 3d). The basal induction of the $h s p-6 p:: g f p$ was quantified as a reporter of the UPR ${ }^{\mathrm{mt}}$ activation by processing the fluorescent images to extract the signal-to-background ratio (SBR). The linear SBR increase over time (Fig. 3e) suggests that the mitochondrial stress pathway is weakly activated in basal conditions $^{29,37}$. The fluorescent signal per worm area at each larval stage (F(L1), F(L2), F(L3), F(L4), F(YA)) was also computed (Fig. 3f).

\section{Phenotypic analysis upon doxycycline treatment}

The platform was used to phenotypically characterize the effect of doxycycline. Cluster analysis (Fig. 4) and principal component analysis (PCA) (Supplementary Fig. S2 and Supplementary Table 1) were first run on the dense datasets to identify features of interest, and provide an overall phenotypic footprint of the effect of doxycycline on the treated worm population. This antibiotic 


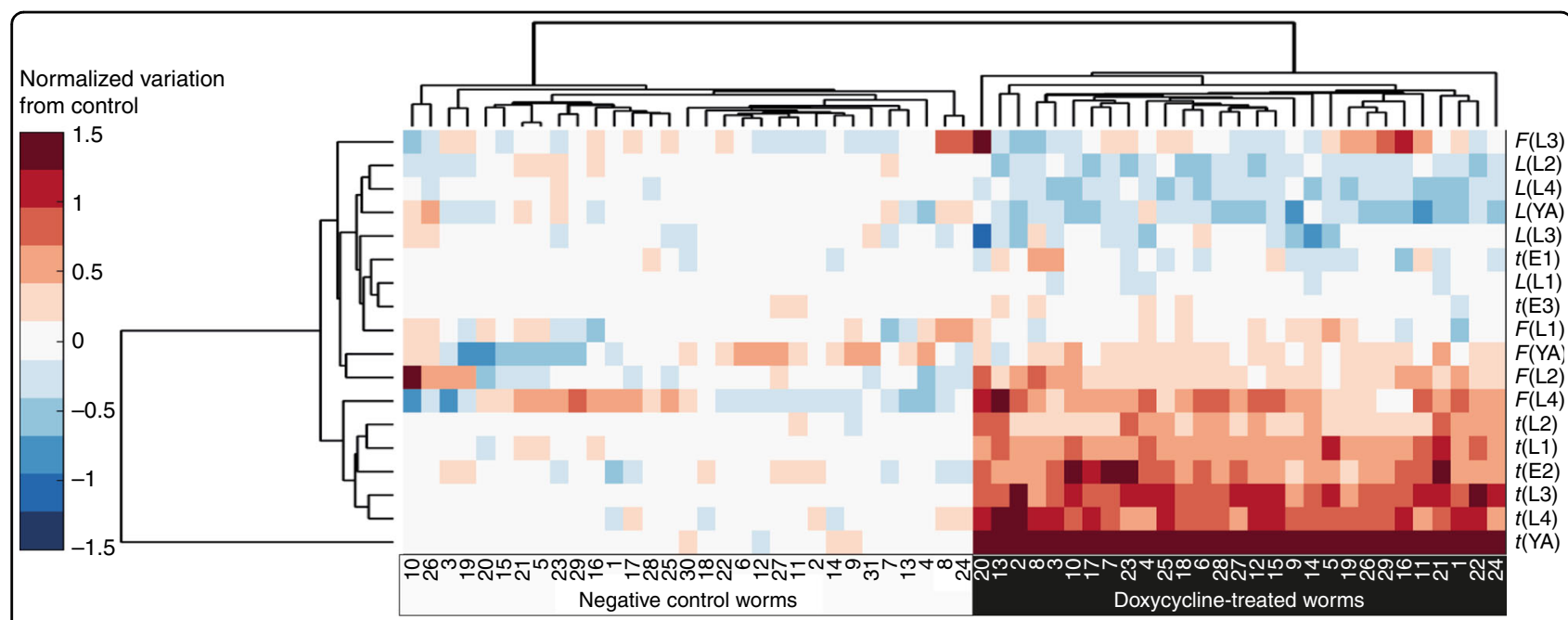

Fig. 4 Hierarchical cluster analysis of phenotypes of the control group and the treated group

induced severe deviations from control, as treated animals showed longer development time $t(\mathrm{~L} 3), \mathrm{t}(\mathrm{L} 4)$ and $\mathrm{t}(\mathrm{YA})$, confirming the doxycycline role in prolonging the animal development and extending its lifespan.

Interestingly, the unexpected permeability of the eggshell to doxycycline induced two different responses on the embryo population. The doxycycline treatment proved to be lethal for $9.4 \%$ of the embryos, that died before hatching (Fig. 5a). This response is in line with the effect of other mitochondrial stressors ${ }^{39,40}$. On the other hand, the embryo development of the surviving eggs was severely delayed (Fig. 5b), especially during the beantwitching phase $t(E 2)$. This effect was also observed in a previous study using a genetic mutation to induce mitochondrial stress ${ }^{25}$. As predicted by cluster analysis, the impact of doxycycline was even more severe on the larval development time (Fig. 5c) and size (Fig. 5d and Supplementary Fig. S3), which featured a 1-fold or higher increase over the negative control, especially for the L4 and the YA stages. This observation agrees with previous reports showing a similar delay in larval development when the electron transport chain function is compromised genetically ${ }^{41}$. Indeed, during the L3-L4 stages, mitochondria undergo a period of dramatic proliferation $^{42}$, making it the critical time-window, during which mitochondrial perturbations can have large impact on C. elegans larval development ${ }^{41}$. Moreover, doxycycline not only impairs gonad development and causes infertility (Fig. 5e), but also pushes $8 \%$ of the individuals towards male development (Fig. 5f). These findings suggest that doxycycline exerts profound effects on developmental processes relying on mitochondrial support, favouring a shift towards male progeny formation, similar to what is observed with other sources of stress ${ }^{43}$, but, interestingly, only in a subgroup of the population. Finally, the tracking of hsp-6p::gfp expression (Fig. 5g, h), showed an overall increase starting at the L2 larval stage, which confirms the induction of mitochondrial stress by doxycycline, followed by the UPR ${ }^{\mathrm{mt}}$.

\section{Discussion}

Quantifying and elucidating the patterns linking markers to heterogeneous responses amongst treated individuals is a paramount prerequisite in precision medicine research. However, this variability in phenome data readout is often lost owing to the lack of specific strategies to tackle this huge challenge. Consequently, we described here a platform that allows culturing and phenotyping of individual worms from a C. elegans population from egg laying until adulthood. Thanks to hydrodynamic trapping, freshly-laid single embryos were automatically isolated and-for the first time-kept in separate chambers until adulthood. The single-worm resolution of our chip allowed the longitudinal monitoring and tracking of multiple phenotypes over the life-cycle of individual organisms. Moreover, worms showed a physiological development consistent with the one in solid medium at the same temperature, thus setting a new standard in terms of $C$. elegans culture and feeding in liquid environment. Therefore, with respect to previous studies on C. elegans culture ${ }^{14,25}$ and its use in drug testing ${ }^{18,25}$, we showed a comprehensive microfluidic platform enabling the unprecedented C. elegans culture over its full life-cycle at the single-worm level while longitudinally monitoring multiple phenotypic markers upon a variety of treatments. In contrast with solid medium approaches, the platform allowed testing a pharmacological treatment by simply injecting the compound of interest into the microfluidic chip from a dedicated inlet, while ensuring perfect control and reproducibility of all the environmental parameters. 


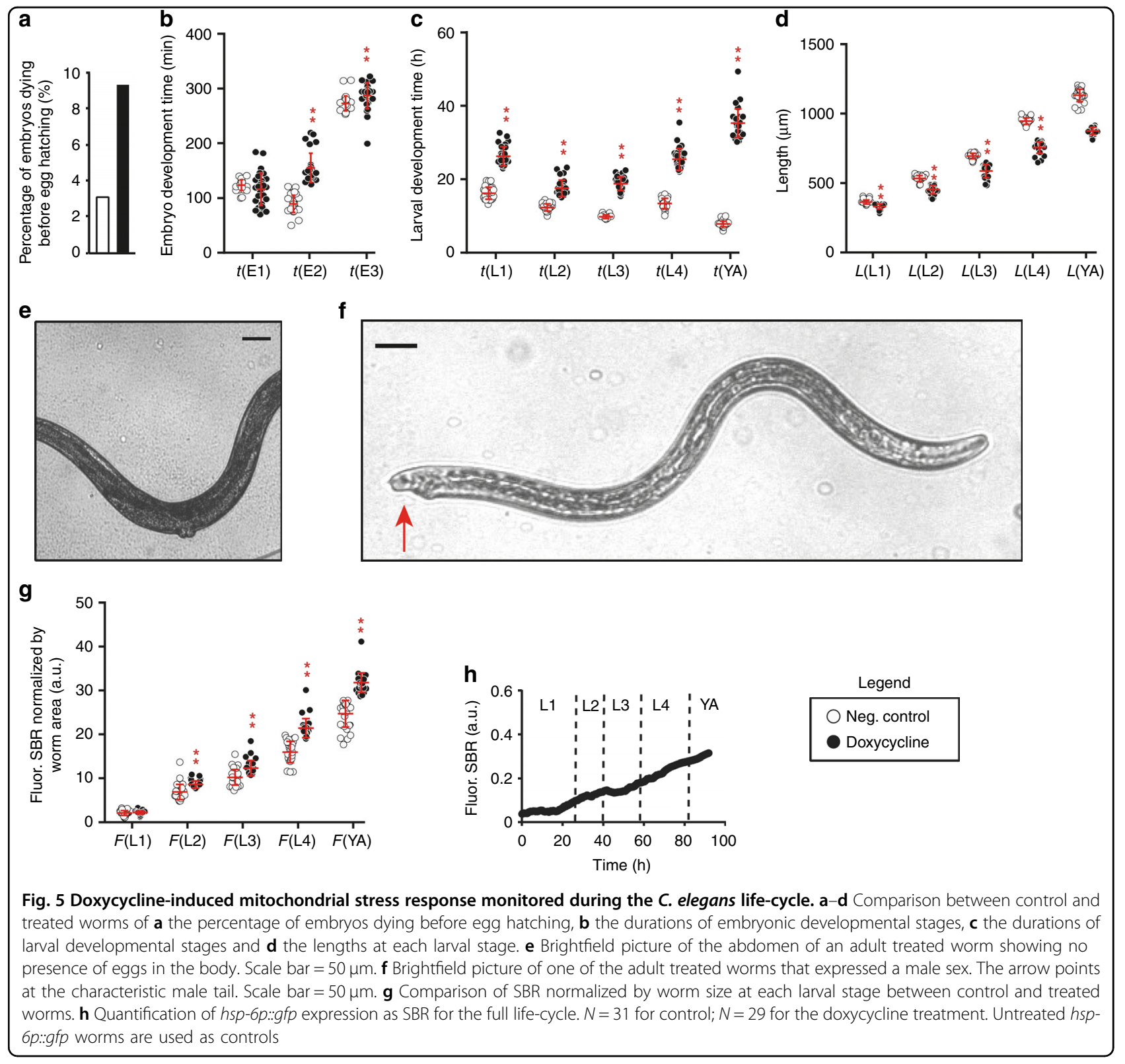

Conjugating longitudinal assays over the full development span with individualized observations in a precisely controlled chemical environment is a clear advancement of the state of the art and a substantial improvement of the previously proposed analytical microfluidic platforms for C. elegans. This phenotyping strategy paves the way for the identification of appropriate disease biomarkers that would shape the worm response to the treatment of interest, which, would complement ideally similar studies performed on other species (reviewed in ref. 44). To compare the high-content phenotypic signature of worm populations under different conditions, we used singleworm based hierarchical clustering analysis and principal component analysis. Big data strategies facilitate the identification of sub-groups and patterns buried in the phenome data. The method also evaluates quantitatively the differences among individuals, and provided the phenotypic footprint of each treatment. Envisioning a personalized medicine in future, the characterization of such intra-population treatment-dependent variability will allow both inferring the effectiveness of a treatment in a population's subsets and identifying personalized follow-up treatments.

The platform and analysis method were used to identify appropriate biomarkers and investigate the phenotypic variability associated with the doxycycline treatment. The classic phenotypic hallmarks of mitochondrial stress were observed, such as delayed development, reduced worm size, reduced fertility and induction of the UPR ${ }^{\mathrm{mt}}$ biomarker $h s p-6 p:: g f p$. However, the setup also revealed a 
surprising treatment-dependent intra-population variability that could otherwise not be observed in experiments run on plates or with different microfluidic culture platforms. Indeed, at the beginning of the life-cycle, doxycycline was lethal to a small group of embryos that could not complete the development, while it prolonged the embryonal development of the others. Moreover, during the gonad development, doxycycline affected the sex fate of a subgroup of embryos that eventually developed as males and not as the usual hermaphrodites. Finally, the fact that the heterogeneous response was observed during both the embryonic and the larval development, confirms the actual need for a microfluidic culture device that allows performing the drug treatment, and monitor its effect, throughout the full animal's life-cycle.

\section{Conclusions}

We presented a versatile microfluidic platform, configured with a modular high-content phenotypic analysis that could include in the future longitudinal analysis over the full life-cycle of phenotypes as diverse as motility, pharyngeal pumping and expression of different reporter genes. This microfluidic-based phenotyping strategy, combined with advanced analysis of the clinical and molecular phenomes, could be used to identify markers related to treatments whose effects are poorly characterized. Moreover, we envision that this platform could be widely employed for identifying single worms as responders or non-responders to a treatment based on their phenotypic footprint. It might also facilitate phenotypic analysis upon RNAi screenings where intrinsically a strong variability is to be expected ${ }^{3}$. Translation of these findings to humans could open breakthroughs in early phases of drug development and medicine through follow-up treatments tailored to the characteristics of the patient.

\section{Acknowledgements}

This work in the M.A.G. laboratory was supported by the Ecole Polytechnique Fédérale de Lausanne and the EU Ideas program (ERC-2012-AdG-320404). V.S. was supported by the 'EPFL Fellows' program co-funded by Marie SkłodowskaCurie, Horizon 2020 Grant agreement (665667). M.C. and L.M. were supported by the Gebert Rüf Stiftung (GRS-025/16). The work in the J.A. laboratory is supported by the EPFL, NIH (R01AG043930), Systems X (SySX.ch 2013/153), Velux Stiftung (1019), and the Swiss National Science Foundation (31003A140780). The authors thank D. Dumont-Fillon for his help during preliminary microfluidic chip design, K. Suter (Center of Microtechnology and Nanotechnology of EPFL) for assistance in the microfabrication processes, T. Lehnert, R. Padovani, D.G. Dupouy (Laboratory of Microsystems LMIS2 at EPFL), and E. Rollo (Laboratory of Life Sciences Electronics CLSE at EPFL) for fruitful discussions about the project.

\section{Authors' contributions}

M. C. L., M. C., J. A. and M. A. M. G. conceived and designed the project. M. C. L. and M. C. designed the platform, M. C. L. fabricated and tested the platform and performed the experiments, L. M. and V. S. provided the worm strains, bacteria and drugs, M. C. L., R. T. and M. S. B. S. performed the data analysis and the statistical analysis, M. C. L. and M. A. M. G. wrote the manuscript with the help of all the authors. All authors read and approved the final manuscript.
M. A. M. G. and M. C. have an International Patent Application $n^{\circ}$ PCT/WO 2016/063199 A1, filed on 20th October 2014 as provisional application $n^{\circ}$ PCT/ IB214/065472, for a device that is related to this work.

\section{Conflict of interest}

M. A. M. G. and M. C. have an International Patent Application $n^{\circ}$ PCT/WO 2016/063199 A1, filed on 20th October 2014 as provisional application $n^{\circ}$ PCT/ IB214/065472, for a device that is related to this work. The remaining authors declare that they have no conflict of interest.

Supplementary information accompanies this paper at https://doi.org/ 10.1038/s41378-018-0003-8.

Received: 3 August 2017 Revised: 22 November 2017 Accepted: 22 December 2017

Published online: 07 May 2018

References

1. Schork, N. J. Personalized medicine: time for one-person trials. Nat. News $\mathbf{5 2 0}$ 609 (2015)

2. Song, P., Hu, R., Tng, D. J. H. \& Yong, K.-T. Moving towards individualized medicine with microfluidics technology. RSC Adv. 4, 11499-11511 (2014).

3. Shapiro, E., Biezuner, T. \& Linnarsson, S. Single-cell sequencing-based technologies will revolutionize whole-organism science. Nat. Rev. Genet. 14, 618-630 (2013)

4. Gupta, B. P. \& Rezai, P. Microfluidic approaches for manipulating, imaging, and screening C. elegans. Micromachines 7, 123 (2016).

5. Muthaiyan Shanmugam, M. \& Subhra Santra, T. Microfluidic devices in advanced Caenorhabditis elegans research. Molecules 21, 8 (2016).

6. San-Miguel, A. \& Lu, H. Microfluidics as a tool for C. elegans research. WormBook 1-19 (2013). https://doi.org/10.1895/wormbook.1.162.1

7. Sivagnanam, V. \& Gijs, M. A. M. Exploring living multicellular organisms, organs, and tissues using microfluidic systems. Chem. Rev. 113, 3214-3247 (2013).

8. Xian, B. et al. WormFarm: a quantitative control and measurement device toward automated Caenorhabditis elegans aging analysis. Aging Cell. 12, 398-409 (2013).

9. Ying, D. et al. A droplet-based microfluidic device for long-term culture and longitudinal observation of Caenorhabditis elegans. BioChip J. 6, 197-205 (2012).

10. Aubry, G., Zhan, M. \& Lu, H. Hydrogel-droplet microfluidic platform for highresolution imaging and sorting of early larval Caenorhabditis elegans. Lab. Chip. 15, 1424-1431 (2015)

11. Shi, W., Qin, J., Ye, N. \& Lin, B. Droplet-based microfluidic system for individual Caenorhabditis elegans assay. Lab. Chip. 8, 1432-1435 (2008).

12. Wen, H., Yu, Y., Zhu, G., Jiang, L. \& Qin, J. A droplet microchip with substance exchange capability for the developmental study of C. elegans. Lab. Chip. 15, 1905-1911 (2015).

13. Clausell-Tormos, J. et al. Droplet-based microfluidic platforms for the encapsulation and screening of mammalian cells and multicellular organisms. Chem. Biol. 15, 427-437 (2008)

14. Gritti, N., Kienle, S., Filina, O. \& van Zon, J. S. Long-term time-lapse microscopy of C. elegans post-embryonic development. Nat. Commun. 7, 12500 (2016).

15. Bringmann, H. Agarose hydrogel microcompartments for imaging sleep- and wake-like behavior and nervous system development in Caenorhabditis elegans lanvae. J. Neurosci. Methods 201, 78-88 (2011).

16. Chung, $K$. et al. Microfluidic chamber arrays for whole-organism behaviorbased chemical screening. Lab. Chip. 11, 3689-3697 (2011).

17. Lockery, S. R. et al. A microfluidic device for whole-animal drug screening using electrophysiological measures in the nematode C. elegans. Lab. Chip. 12, 2211-2220 (2012)

18. Mondal, S. et al. Large-scale microfluidics providing high-resolution and highthroughput screening of Caenorhabditis elegans poly-glutamine aggregation model. Nat. Commun. 7, 13023 (2016).

19. Hulme, S. E. et al. Lifespan-on-a-chip: microfluidic chambers for performing lifelong observation of C. elegans. Lab. Chip. 10, 589-597 (2010).

20. Krajniak, J. \& Lu, H. Long-term high-resolution imaging and culture of C. elegans in chip-gel hybrid microfluidic device for developmental studies. Lab. Chip. 10, 1862-1868 (2010). 
21. Li, S., Stone, H. A. \& Murphy, C. T. A microfluidic device and automatic counting system for the study of $\mathrm{C}$. elegans reproductive aging. Lab. Chip. 15 524-531 (2014).

22. Akagi, J. et al. Miniaturized embryo array for automated trapping, immobilization and microperfusion of zebrafish embryos. PLoS One 7, e36630 (2012).

23. Khoshmanesh, $\mathrm{K}$. et al. New rationale for large metazoan embryo manipulations on chip-based devices. Biomicrofluidics 6, 24102-2410214 (2012).

24. Khoshmanesh, K. et al. Trapping and imaging of micron-sized embryos using dielectrophoresis. Electrophoresis 32, 3129-3132 (2011).

25. Cornaglia, M. et al. An automated microfluidic platform for $C$. elegans embryo arraying, phenotyping, and long-term live imaging. Sci. Rep. 5, 10192 (2015).

26. Durieux, J., Wolff, S. \& Dillin, A. The cell-non-autonomous nature of electron transport chain-mediated longevity. Cell 144, 79-91 (2011).

27. Jovaisaite, V., Mouchiroud, L. \& Auwerx, J. The mitochondrial unfolded protein response, a conserved stress response pathway with implications in health and disease. J. Exp. Biol. 217, 137-143 (2014).

28. Lin, Y.F. \& Haynes, C. M. Metabolism and the UPR(mt). Mol. Cell. 61, 677-682 (2016).

29. Houtkooper, R. H. et al. Mitonuclear protein imbalance as a conserved longevity mechanism. Nature 497, 451-457 (2013).

30. Haynes, C. M., Yang, Y., Blais, S. P., Neubert, T. A. \& Ron, D. The matrix peptide exporter HAF-1 signals a mitochondrial UPR by activating the transcription factor ZC376.7 in C. elegans. Mol. Cell. 37, 529-540 (2010).

31. Xia, Y. \& Whitesides, G. M. Soft lithography. Angew. Chem. Int. Ed. 37, 550-575 (1998).

32. Luk, V. N., Mo, G. C. \& Wheeler, A. R. Pluronic additives: a solution to sticky problems in digital microfluidics. Langmuir 24, 6382-6389 (2008).
33. Moullan, N. et al. Tetracyclines disturb mitochondrial function across eukaryotic models: a call for caution in biomedical research. Cell Rep. 10, 1681-1691 (2015).

34. Stiernagle, T. Maintenance of C. elegans. WormBook (2006). https://doi.org/ 10.1895/wormbook.1.101.1

35. Cassada, R. C. \& Russell, R. L. The dauerlarva, a post-embryonic developmental variant of the nematode Caenorhabditis elegans. Dev. Biol. 46, 326-342 (1975).

36. Schierenberg, E. Embryological variation during nematode development. WormBook (2006). https://doi.org/10.1895/wormbook.1.55.1

37. Yoneda, T. et al. Compartment-specific perturbation of protein handling activates genes encoding mitochondrial chaperones. J. Cell. Sci. 117 4055-4066 (2004).

38. Corsi, A. K., Wightman, B. \& Chalfie, M. A transparent window into biology: a primer on Caenorhabditis elegans. Genetics 200, 387-407 (2015).

39. Avery, L. The genetics of feeding in Caenorhabditis elegans. Genetics 133 897-917 (1993).

40. Haynes, C. M., Petrova, K., Benedetti, C., Yang, Y. \& Ron, D. ClpP mediates activation of a mitochondrial unfolded protein response in C. elegans. Dev. Cell. 13, 467-480 (2007).

41. Dillin, A. et al. Rates of behavior and aging specified by mitochondrial function during development. Science 298, 2398-2401 (2002).

42. Tsang, W. Y. \& Lemire, B. D. Mitochondrial genome content is regulated during nematode development. Biochem. Biophys. Res. Commun. 291, 8-16 (2002).

43. Wood, W. B., Sulston, J. \& Hodgkin, J. The Nematode Caenorhabditis elegans. Cold Spring Harbor Laboratory, New York (1988).

44. Williams, E. G. \& Auwerx, J. The convergence of systems and reductionist approaches in complex trait analysis. Cell 162, 23-32 (2015). 